\title{
QUALITY FACTOR ISSUES IN SILICON CARBIDE NANOMECHANICAL RESONATORS
}

\author{
X.M.H. Huang ${ }^{1, *}$, C.A. Zorman ${ }^{2}$, M. Mehregany ${ }^{2}$ \& M.L. Roukes ${ }^{1}$ \\ ${ }^{1}$ Condensed Matter Physics, California Institute of Technology, Pasadena, California 91125 \\ ${ }^{2}$ Electrical Engineering and Computer Science, Case Western Reserve University, Cleveland, OH 44106
}

\begin{abstract}
Nanomechanical resonators with fundamental mode resonance frequencies in the Very-High Frequency (VHF), Ultra-High Frequency (UHF) range and microwave L-band are fabricated from monocystalline silicon carbide thin film material, and measured by magnetomotive transduction, combined with a balanced bridge read out circuit. For resonators made from the same film, we measured the frequency (i.e., geometry) dependence of the quality factor. It is found that the quality factor of these resonators decreases when the frequency increases. This indicates the importance of clamping loss in this regime. In addition, from studies of resonators made from different chips with varying surface roughness, we found a strong correlation between surface roughness of the silicon carbide thin film material and the quality factor of the resonators made from it. Understanding the dissipation mechanisms, and thus improving the quality factor of these resonators, is important for implementing applications promised by these devices.
\end{abstract}

\section{INTRODUCTION}

Nanoelectromechanical Systems (NEMS) with frequencies entering microwave L-band have recently been achieved $^{1}$, owing to the parallel development of better materiais $^{2}$ and novel detection techniques ${ }^{3}$. This new breakthrough promises a broad range of applications, including next generation high resolution sensors and actuators, and high speed signal processing components ${ }^{4,5,6,7,8}$. These devices also offer the potential advantage of much greater integratability over what is currently available. However, resonators with fundamental frequencies in the $\mathrm{UHF} /$ microwave range have exhibited deteriorating quality factors compared to devices at lower frequencies, which could significantly restrict the application of this developing technology. This paper summarizes our initial attempt to address this important problem, as well as our efforts to improve the measurement techniques at these frequencies.

\section{EXPERIMENTAL DETAILS}

3C-SiC nanomechanical resonators were fabricated using epitaxial growth and e-beam lithography techniques detailed elsewhere ${ }^{2}$. Here, we discuss the recipe optimized for devices designed to operate at frequencies above 100 MHz.

Briefly, the 3C-SiC films used in this work are heteroepitaxially grown on $100 \mathrm{~mm}$-diameter (100) $\mathrm{Si}$

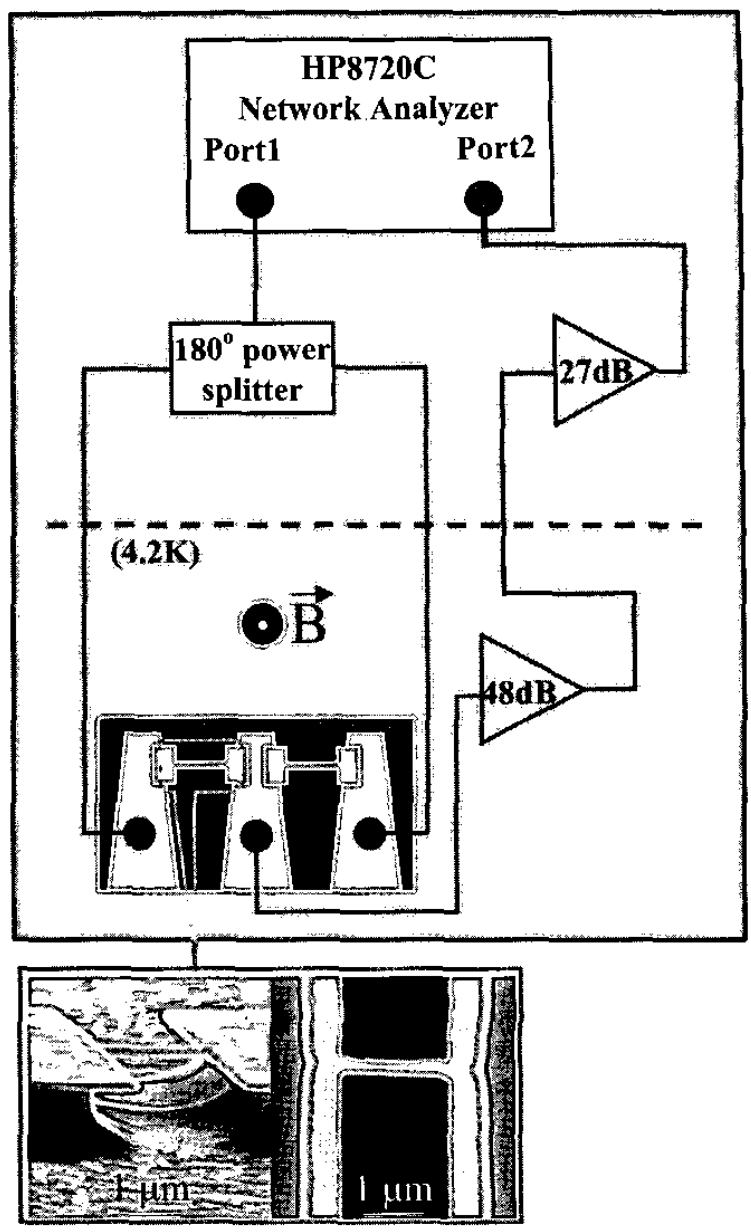

Figure 1. Schematic circuit diagram for balanced electronic detection. (call-out box) Side and top zoom-in view of one of the two doubly clamped beams in the device structure.

wafers in a rf-induction-heated, atmospheric pressure chemical vapor deposition reactor. $\mathrm{SiH}_{4}$ and $\mathrm{C}_{3} \mathrm{H}_{8}$ are used as precursors, and $\mathrm{H}_{2}$ is used as a carrier gas. The epitaxial process is a two-step, high-temperature $\left(1280^{\circ} \mathrm{C}\right)$ procedure, involving the carbonization of the $\mathrm{Si}$ surface in a $\mathrm{C}_{3} \mathrm{H}_{8} / \mathrm{H}_{2}$ ambient followed by epitaxial growth using $\mathrm{SiH}_{4}$, $\mathrm{C}_{3} \mathrm{H}_{8}$, and $\mathrm{H}_{2}$. Device fabrication utilizes a combination of optical and electron beam lithography techniques. The process begins by using standard photoresist and optical

* Presenting Author: X.M.H.Huang, e-mail address xmhenryhuang@caltech.edu, Ph (626) 688-0317, FAX (626) 683-9060

TRANSDUCERS ' 03

The 12th International Conference on Solid State Sensors, Actuators and Microsystems, Boston, June 8-12, 2003 


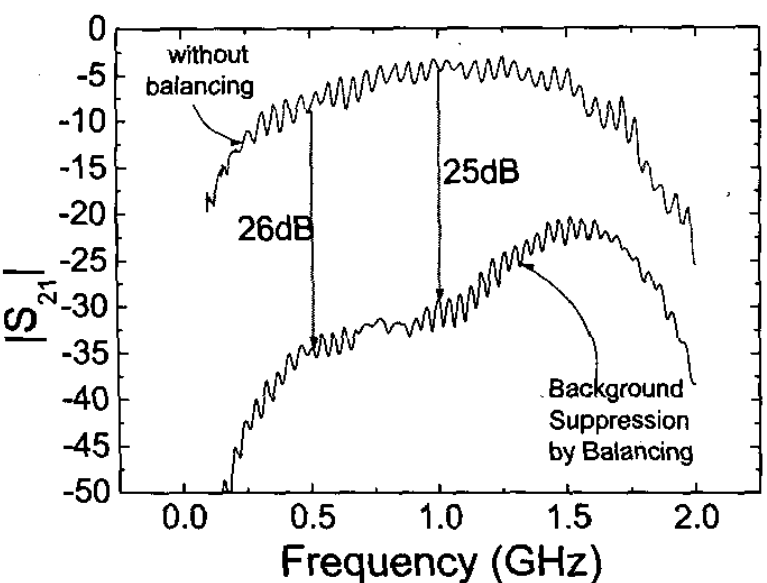

Figure 2. Suppression of detection background by the balancing technique.

lithography to define the large-area contact pads which consist of a $6 \mathrm{~nm}$-thick $\mathrm{Cr}$ adhesion layer and a $80 \mathrm{~nm}$-thick $\mathrm{Au}$ film. The substrates are then coated with a PMMA thin film, which is then patterned by electron beam lithography into a metallic lift-off mold that defines the submicron mechanical components of the $\mathrm{SiC}$ devices. The patterned metal mask is transferred to the $3 \mathrm{C}-\mathrm{SiC}$ layer by electron cyclotron resonance (ECR) etching using a $\mathrm{NF}_{3} / \mathrm{O}_{2} / \mathrm{Ar}$ anisotropic etch. The newly patterned 3C-SiC beams are then released by simply etching the underlying Si substrate using an isotropic $\mathrm{NF}_{3} / \mathrm{Ar}$ ECR etch. The metallic etch mask remains on the $\mathrm{SiC}$ beams to be used as a conducting layer for device testing.

Here, we employ $30 \mathrm{~nm}$ of $\mathrm{Al}$, followed by $5 \mathrm{~nm}$ of $\mathrm{Ti}$ as the choice for device metallization, even though other metals such as. $\mathrm{Au}$ and $\mathrm{Ni}$ are also capable of surviving the ECR etching process and can provide the electrical conductivity required by the detection scheme discussed later in this paper. The low mass density of $\mathrm{Al}$ helps in reducing mass loading, thus resulting in a higher frequency for the same device geometry as compared with a more dense metal.

A typical suspended nanostructure is shown in the insert of Fig. 1. Two nearly-identical suspended beams form a device structure for testing. Typical devices are about $75 \mathrm{~nm}$ thick, with beam widths of 100 to $150 \mathrm{~nm}$ and lengths of 1.0 to $2.0 \mu \mathrm{m}$. A typical metallized beam has a measured resistance of about $100 \mathrm{Ohm}$ at room temperature, with the resistance mismatch between the two beams in the same device to be within a few percent.

Device testing is performed in ultrahigh vacuum (provided by liquid helium cooling of a sealed dipper) and involves the use of magnetomotive transduction ${ }^{9,10}$ in concert with a variation of the balancing technique ${ }^{3}$ tailored for $\mathrm{VHF} / \mathrm{UHF} / \mathrm{Microwave}$ applications. The schematic

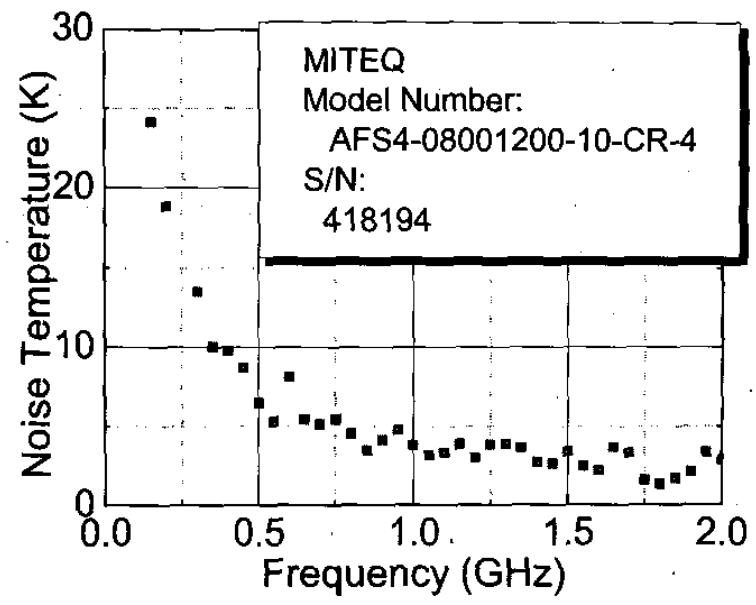

Figure 3. Measured noise temperature of the cryogenicamplifier when it is immersed in liquid helium.
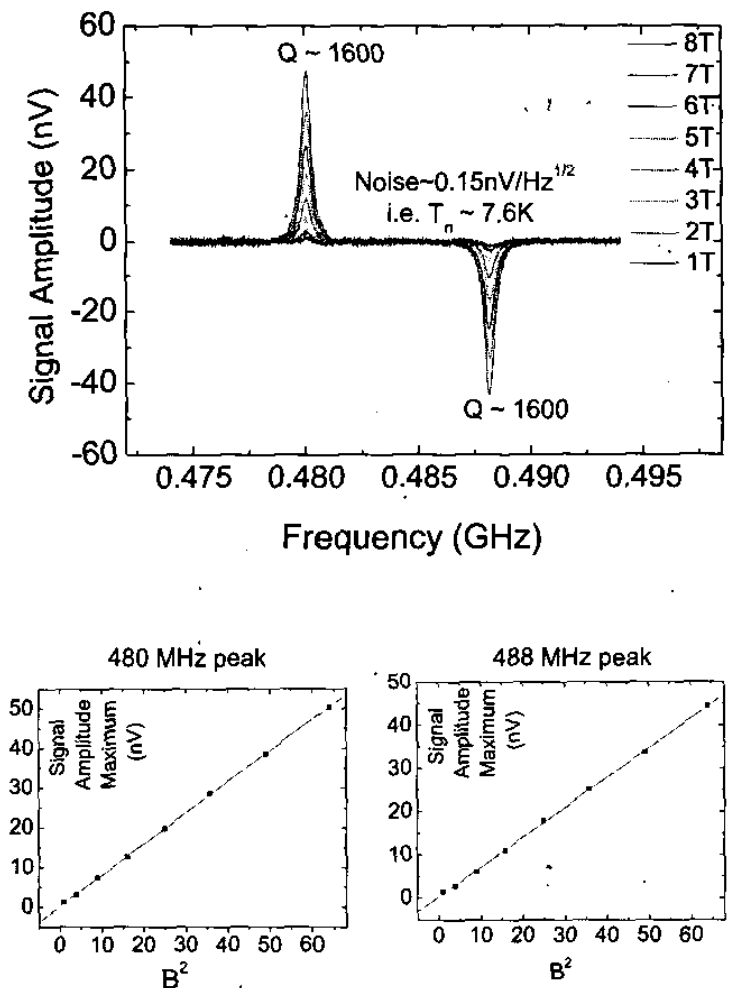

Figure 4. (upper) Typical UHF resonance curves at different magnetic fields measured using the circuit shown in Fig. 1. (lower) The two figures below show that the signal amplitude is proportional to $B^{2}$, as expected.

TRANSDUCERS ' 03

The 12th International Conference on Solid State Sensors, Actuators and Microsystems, Boston, June 8-12, 2003 
circuit diagram is shown in Fig. 1. The sample is positioned in a magnetic field with the beams perpendicular to the field lines. The potential of the center pad is initially held at virtual ground by using a 180 degree power splitter to provide out-of-phase driving of the two device branches and by making the two branches of the circuitry as close to identical as possible. The two beams are also nearly (but not exactly) identical to each other. As a result, they have slightly different resonance frequencies. When the drive frequency is swept to match the fundamental resonance frequency of one of the beams, resonant motion is induced to cut the field lines resulting in an emf voltage that can be detected. Nonidealities in the system produce a high residual background, which can be reduced using the balancing technique (Fig. 2). The improvement here is limited by the resistance mismatch (of a few percent) in between the two beams, which was sufficient for this experiment. Further improvement can be made by using variable attenuators and adjustable phase shifters to further match the two braches of the driving circuit. Use of a low noise cryogenic amplifier (Fig. 3) enables the detection of displacements on the order of femtometers. Resonance curves at different $B$ fields for a typical UHF resonator pair are shown in Fig. 4. The peak and the dip represent the resonance of each of the two doubly-clamped beams in the device structure. The observed noise temperature (referred back to the input of the amplifier) is about $7.6 \mathrm{~K}$. The noise comes from a combination of amplifier noise and the Johnson noise due to beam resistance sitting at $4.2 \mathrm{~K}$. The noise temperature implies that a displacement detection sensitivity on the order of femtometers $/ \mathrm{Hz}^{1 / 2}$ has been achieved in our experiments. The maximum signal amplitude depends linearly on $\mathrm{B}^{2}$, as expected: The peaks are fitted to a Lorentzian curve in order to extract the quality factor, $\mathrm{Q}$. Here, we adopt the definition most popularly used in the field, namely, $Q=\omega_{0} / \Delta \omega$, where $\omega_{0}$ is the resonance frequency, $\Delta \omega$ is peak width in power spectrum (i.e., width at $1 / 2^{1 / 2}$ maximum signal amplitude), though another convention, which uses full-width at half amplitude as peak width, also appears in the literature (such as. Ref. 2). The two definitions differ only by a numerical constant, with the $Q$ value following the latter definition being smaller by a factor of $3^{1 / 2}$.

The frequency dependence of $\mathrm{Q}$ for a family of devices fabricated from the same 3C-SiC film is plotted in Fig. 5 . The beams were identical in nearly every parameter except length, with the shortest beams $(1.1 \mu \mathrm{m})$ yielding the highest resonant frequencies. The steady decline in $Q$ with increasing frequency for beams made from the same film suggests that a clamping loss mechanism may influence $Q$ at $\mathrm{VHF} / \mathrm{UHF} / \mathrm{microwave}$ frequencies.

Two approaches (or their combination) may be used to alleviate clamping loss at such frequencies. One is the application of a free-free beam design ${ }^{11,12}$, to clamp the beams at the motional nodal points of fundamental resonance, and strategically use a quarter wavelength

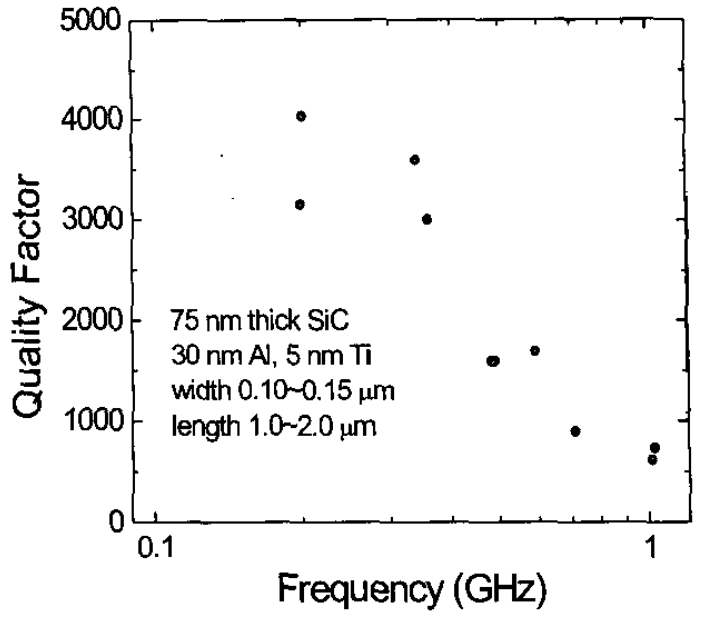

Figure 5. Quality factor vs. Frequency plot for doublyclamped beam devices made out of the center area of Wafer B (see Fig.6). These devices differ only in e-beam writing pattern geometry. The plot shows a gradual decrease in $Q$ when the device size becomes smaller, i.e. frequency becomes higher.

support structure to minimize energy transfer from the resonance mode to the environment. The other is to further shrink the size of the devices. For the same operating frequency, a smaller width along the direction of motion would allow larger aspect ratio to be used, thus reducing clamping loss and improving $\mathrm{Q}$, according to the results we showed here.

To investigate the effect of film quality on performance, devices were fabricated from films that differed significantly in surface roughness by fabricating the devices on chips from different wafers (see Fig. 6). In all cases, devices operational in the UHF/microwave regime were made from films that had a low surface roughness ( $2.1 \mathrm{~nm}$ ). In contrast, devices made from rougher films (up to $\sim 7.1 \mathrm{~nm}$ ) were operational into the VHF range, but not higher. For our setup, failure to detect any signal indicates an upper limit in the quality factor of the non-operational structures to be well below $\sim 100$. These results suggest a strong correlation between quality factor and surface roughness.

The epitaxial growth recipe used to prepare the $3 \mathrm{C}-\mathrm{SiC}$ films was initially designed for MEMS applications and thus was optimized for micron-thick films yet can produce $50 \mathrm{~nm}$ to $250 \mathrm{~nm}$-thick films of sufficient surface quality for e-beam lithography. Our results indicate that while the surfaces might be sufficient for fabrication purposes, it is critically important that the growth processes be optimized to produce ultrasmooth $\mathrm{SiC}$ thin films if these films are to be used for nanomechanical resonators having reasonably high $Q$ values. The issue of surface roughness is only now coming to the forefront, since up to recently, 


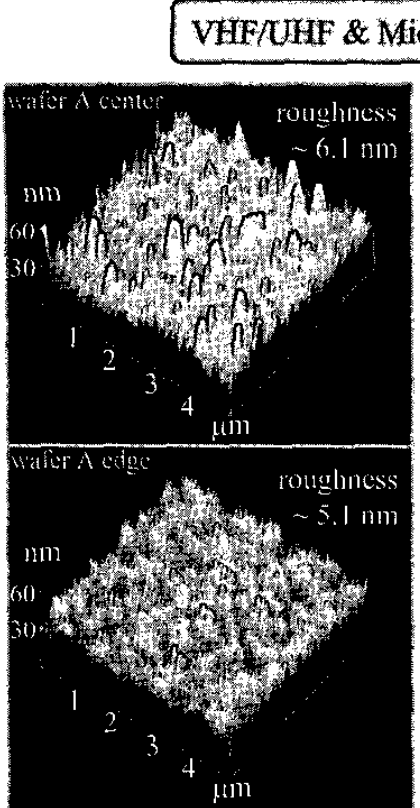

Wafer A $(\sim 50 \mathrm{~nm} \mathrm{SiC})$ \#99953035UN3.00B0C4

\section{owave NEMS}

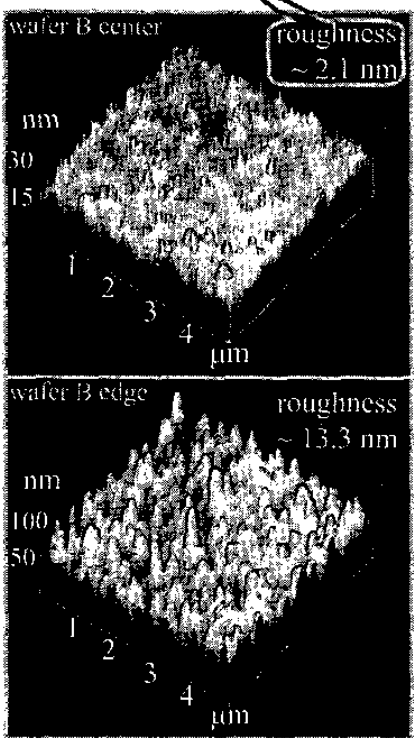

Wafer B $(\sim 75 \mathrm{~nm} \mathrm{SiC})$ \#SICLPOCWRU0699-263

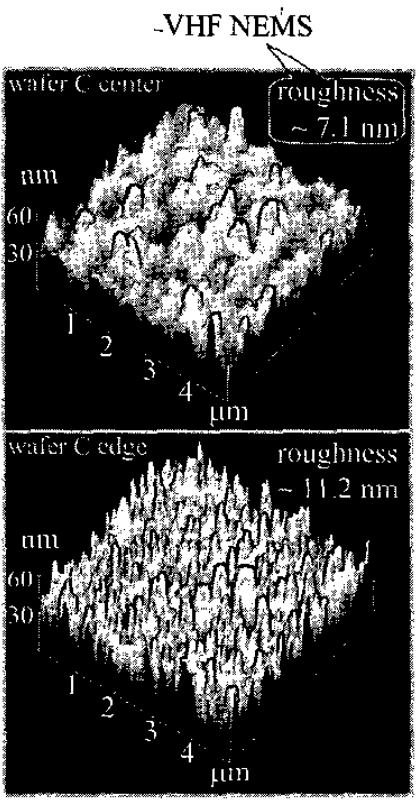

Wafer C $(\sim 200 \mathrm{~nm} \mathrm{SiC})$ \#086SKBAGUN05

Figure 6. AFM images of surface morphology of three SiC films, labeled A, B\& C. It is observed that all successfully measured UHF/Microwave devices are fabricated from the center area of wafer $B$. Center area of wafer $C$ has produced some good devices in VHF frequency range, but fails to work in the UHF and microwave frequency range.

nanomechanical beams have only been fabricated from $\mathrm{Si}$ wafers (bulk and SOI) and epitaxial III-V films (i.e. GaAs) that have ultrasmooth surfaces by virtue of a long history in microelectronics. And this issue will continue to be important as new materials are added to the NEMS toolbox in response to the widening array of applications. Recipes for growing ultrathin SiC films optimized for surface smoothness, and techniques for $\mathrm{SiC}$ thin film surface polishing are currently under development.

\section{CONCLUSIONS}

In conclusion, we have demonstrated that the resonant frequency of silicon carbide nanomechanical resonators designed to operate in the UHF to low microwave L-band range can be measured using magnetomotive transduction techniques. Dissipation in such devices increases as the aspect ratio of the doubly-clamped beams is reduced. A strong correlation between surface roughness and deteriorating quality factor is established from our experiments. Understanding the dissipation mechanisms and achieving the ability to improve the quality factor of such resonators is crucial for future NEMS applications.

Acknowledgements: This work was generously supported by DARPA MTO/MEMS and NSF.

\section{REFERENCES}

${ }^{\prime}$ X. M. H. Huang, C. A. Zorman, M. Mehregany \& M. L. Roukes, "Nanodevice motion at microwave frequencies", Nature, 421, 496 (2003).

${ }^{2}$ Y. T. Yang, K. L. Ekinci, X. M. H. Huang, L. M. Schiavone, M. L. Roukes, C. A. Zorman, \& M. Mehregany, "Monocrystalline silicon carbide nanoelectromechanical systems", Appl. Phys. Lett., 78(2), 162-164 (2001).

${ }^{3}$ K. L. Ekinci, Y. T. Yang, X. M. H. Huang, \& M. L. Roukes, "Balanced electronic detection of displacement in nanoelectromechanical systerns", Appl. Phys, Lett., 81(12), 2253-2255 (2002).

${ }^{4}$ M.L. Roukes, "Plenty of Room, Indeed", Scientific American 285, pp. 48-57 (2001)

${ }^{5}$ M.L. Roukes, "Nanomechanical Systems face the future", Physics World 14, pp. 25-31 (2001).

${ }^{6}$ A.N. Cleland, M.L. Roukes, "A nanometer-scale mechanical electrometer", Nature 392, 160-162 (1998).

${ }^{7}$ C.T.C. Nguyen, L.P.B. Katehi, G.M. Rebeiz, "Micromachined devices for wireless communications", Proceedings of the IEEE $86(8)$ : 1756-1768 (1998).

${ }^{8}$ D. W. Car, S. Evoy, L. Sekaric, H. G. Craighead and J. M. Parpia, "Measurement of mechanical resonance and losses in nanometer scale silicon wires", Appl. Phys. Lett., 75, 920-922 (1999).

${ }^{9}$ A.N. Cleland and M.L. Roukes, "Fabrication of high frequency nanometer scale mechanical resonators from bulk Si crystals", Appl. Phys. Lett. 69, 2653-2655 (1996).

. ${ }^{10}$ A.N. Cleland and M.L. Roukes, "External control of dissipation in a nanometer-scale radiofrequency mechanical resonator", Sensors and Actuators, 72, 256-261 (1999).

${ }^{11} \mathrm{~K}$. Wang, Y. Yu, A.-C. Wong, and C. T.-C. Nguyen, "VHF free-free beam high-Q micromechanical resonators", Technical Digest, 12th International IEEE Micro Electro Mechanical Systerns Conference, Orlando, Florida, Jan. 17-21, 1999, pp. 453-458.

${ }^{12}$ W. -T. Hsu, J. R. Clark, and C. T. -C. Nguyen, " $Q$-optimized lateral free-free beam micromechanical resonators", Digest of Technical Papers, the $11^{\text {th }}$ Int. Conf. on Solid-State Sensors \& Actuators (Transducers'01), Munich, Germany, June 10-14, 2001, pp. 1110-1113.

\section{TRANSDUCERS '03}

The 12th International Conference on Solid State Sensors, Actuators and Microsystems, Boston, June 8-12, 2003 